\title{
RIGIDITY OF STATIONARY BLACK HOLES WITH SMALL ANGULAR MOMENTUM ON THE HORIZON
}

\author{
S. ALEXAKIS, A. D. IONESCU, AND S. KLAINERMAN
}

\begin{abstract}
We prove a black hole rigidity result for slowly rotating stationary solutions of the Einstein vacuum equations. More precisely, we prove that the domain of outer communications of a regular stationary vacuum is isometric to the domain of outer communications of a Kerr solution, provided that the stationary Killing vector-field $\mathbf{T}$ is small on the bifurcation sphere, i.e. the corresponding Black Hole has small angular momentum. No other global restrictions are necessary. The proof brings together ideas from our previous work [1 with ideas from the classical work of Sudarsky and Wald 17. on the staticity of stationary black hole solutions with zero angular momentum on the horizon. It is thus the first uniqueness result, in the framework of smooth, asymptotically flat, stationary solutions, which combines local considerations near the horizon, via Carleman estimates, with information obtained by global elliptic estimates.
\end{abstract}

\section{Contents}

1. Introduction

1.1. Main Theorem

1.2. The precise quantitative assumptions

2. Proof of the main theorem

2.1. Some useful identities.

2.2. Proof of Proposition 1.3

References

\section{INTRODUCTION}

In this paper we prove a new black hole rigidity result for slowly rotating stationary solutions of the Einstein vacuum equations. More precisely, we show that the domain of outer communications of any smooth stationary regular vacuum black hole with the stationary Killing vector-field $\mathbf{T}$ being small on the bifurcation sphere of the horizon must be isometric to the domain of outer communications of a Kerr solution $K(a, M)$ with small angular momentum $a / M$. This should be compared with our previous result

The first author was partially supported by NSERC grants 488916 and 489103, and a Sloan fellowship. The second author was partially supported by a Packard Fellowship. The third author was partially supported by NSF grant DMS-2156449. All authors were also supported by NSF-FRG grant DMS1065710. 
in [2] in which rigidity was proved, for the entire range $0 \leq a<M$, under a global smallness assumption on the Mars-Simon tensor associated to the stationary space-time. That result rested on three important ingredients:

(1) An unconditional local rigidity result, established in [1] (see also [12 ) according to which a second, rotational Killing vector-field $\mathbf{Z}$ can be constructed in a small neighborhood of the bifurcate sphere of the horizon.

(2) An extension argument for the Killing vector-field $\mathbf{Z}$ based on a global foliation of the space-time with $\mathbf{T}$ - conditional pseudo-convex hypersurfaces. The crucial T- conditional pseudo-convexity condition is ensured by the assumed smallness of the Mars-Simon tensor.

(3) Once $\mathbf{Z}$ is globally extended, and thus the space-time is shown to be both stationary and axisymmetric, one can appeal to the classical Carter-Robinson theorem to conclude the desired rigidity.

The result we present here is still based on the first and third ingredients above but replaces the second one with a new ingredient inspired from the classical work of Sudarsky and Wald [17] (see also [4]) on the staticity of stationary, axially symmetrid], black hole solutions with zero angular momentum. Their result was based on a simple integral formula linking the total extrinsic curvature of a regular maximal hypersurface $\Sigma$ imbedded in the space-time and passing through the bifurcate sphere, with the angular momentum of the horizon. It can be easily shown 2 that zero ADM angular momentum implies vanishing angular momentum of the horizon and thus, in view of the above mentioned formula, the maximal hyper-surface has to be totally geodesic. This then implies the desired conclusion of [17, i.e the space-time is static. The main observation of our result here is that a simple smallness assumption of $\mathbf{T}$ on the bifurcate spher ${ }^{3}$ implies the smallness of the total curvature of the maximal hypersurface. This can then be combined with a simple application of the classical Hopf Lemma to conclude that the entire ergo-region of the black hole can be covered by the local neighborhood of the horizon in which the second, rotational, Killing vector-field $\mathbf{Z}$ has been extended, according to step (1) above. Away from the ergo-region $\mathbf{T}$ is time-like and thus $\mathbf{T}$-conditional pseudo-convexity is automatically satisfied. Thus, the second Killing vector-field $\mathbf{Z}$ can be easily extended to the entire space-time by the results of [10], [11], [2]. Alternatively, since $\mathbf{T}$ is time-like in the complement of the ergo-region, the metric must be real analytic in appropriate coordinates, see [14]. The extension of $\mathbf{Z}$ can then be simply done using the classical results of [15].

1.1. Main Theorem. Our result depends on four types of assumptions.

\footnotetext{
${ }^{1}$ The result assumes in fact analyticity of the space-time which, according to the well known result of Hawking, implies axisymmetry.

${ }^{2}$ This step is based on the assumption of axial symmetry.

3 This is equivalent with a small angular momentum assumption on the horizon. It remains open whether this condition can be replaced with a smallness assumption of the ADM angular momentum.
} 
(1) Standard global regularity and asymptotic flatness assumptions concerning the stationary space-time $(\mathbf{M}, \mathbf{g})$.

(2) Assumptions on the non-degeneracy of the horizon.

(3) Assumptions on the existence and regularity of an asymptotically flat maximal hypersurface passing through the bifurcate sphere of the horizon.

(4) Smallness of the stationary Killing vectorfield $\mathbf{T}$ on the bifurcate sphere of the horizon.

1.1.1. Main Objects. We assume that $(\mathbf{M}, \mathbf{g})$ is a smooth vacuum Einstein space-time of dimension $3+1$ and $\mathbf{T} \in T(\mathbf{M})$ is a smooth Killing vector-field on $\mathbf{M}$. We also assume that we are given an embedded partial Cauchy surface $\Sigma^{0} \subseteq \mathbf{M}$ and a diffeomorphism $\Phi_{0}: E_{1 / 2} \rightarrow \Sigma^{0}$, where $E_{r}=\left\{x \in \mathbb{R}^{3}:|x|>r\right\}$. Moreover, we assume that

$$
\Sigma_{1}:=\Phi_{0}\left(E_{1}\right) \text { is a maximal hypersurface. }
$$

The existence of asymptoticaly flat maximal surfaces with $\partial \Sigma_{1}=S S_{0}$ in stationary spacetimes has been derived in [6] (see theorem 4.2.). The required smoothness and decay at spatial infinity that we assume below can be proved by elliptic estimates; these are however not the purpose of this paper, so we include them as assumptions.

1.1.2. Main regularity assumptions. The regularity assumptions on our space-time, the stationary Killing field, and the bifurcate event horizon are precisely as in [2]. The first assumption is a standard asymptotic flatness assumption which, in particular, defines the asymptotic region $\mathbf{M}^{(e n d)}$ and the domain of outer communications (exterior region) $\mathbf{E}=\mathcal{I}^{-}\left(\mathbf{M}^{(e n d)}\right) \cap \mathcal{I}^{+}\left(\mathbf{M}^{(e n d)}\right)$. Our second assumption concerns the smoothness of the two achronal boundaries $\delta\left(\mathcal{I}^{-}\left(\mathbf{M}^{(e n d)}\right)\right)$ in a small neighborhood of their intersection $S_{0}=$ $\delta\left(\mathcal{I}^{-}\left(\mathbf{M}^{(e n d)}\right)\right) \cap \delta\left(\mathcal{I}^{+}\left(\mathbf{M}^{(e n d)}\right)\right)$. Though this second assumption is not directly used here it was very important in the local construction of a rotational Killing vector-field in [1], see Theorem 1.2 below.

GR. (Global regularity assumption) We assume that the restriction of the diffeomorphism $\Phi_{0}$ to $E_{R_{0}}$, for $R_{0}$ sufficiently large, extends to a diffeomorphism $\Phi_{0}: \mathbb{R} \times$ $E_{R_{0}} \rightarrow \mathbf{M}^{(e n d)}$, where $\mathbf{M}^{(e n d)}$ (asymptotic region) is an open subset of $\mathbf{M}$. In local coordinates $\left\{x^{0}, x^{i}\right\}$ defined by this diffeomorphism, we assume that $\mathbf{T}=\partial_{0}$ and, with $r=\sqrt{\left(x^{1}\right)^{2}+\left(x^{2}\right)^{2}+\left(x^{3}\right)^{2}}$, that the components of the space-time metric verify

$$
\mathbf{g}_{00}=-1+\frac{2 M}{r}+O_{6}\left(r^{-2}\right), \quad \mathbf{g}_{i j}=\delta_{i j}+O_{6}\left(r^{-1}\right), \quad \mathbf{g}_{0 i}=-\epsilon_{i j k} \frac{2 S^{j} x^{k}}{r^{3}}+O_{6}\left(r^{-3}\right),
$$

for some $M>0, S^{1}, S^{2}, S^{3} \in \mathbb{R}$ such that,

$$
J=\left[\left(S^{1}\right)^{2}+\left(S^{2}\right)^{2}+\left(S^{3}\right)^{2}\right]^{1 / 2} \in\left[0, M^{2}\right) .
$$

\footnotetext{
${ }^{4} \mathbf{M}$ is a connected, oriented, time oriented, paracompact $C^{\infty}$ manifold without boundary.

${ }^{5}$ We denote by $O_{k}\left(r^{a}\right)$ any smooth function in $\mathbf{M}^{(e n d)}$ which verifies $\left|\partial^{i} f\right|=O\left(r^{a-i}\right)$ for any $0 \leq i \leq k$ with $\left|\partial^{i} f\right|=\sum_{i_{0}+i_{1}+i_{2}+i_{3}=i}\left|\partial_{0}^{i_{0}} \partial_{1}^{i_{1}} \partial_{2}^{i_{2}} \partial_{3}^{i_{3}} f\right|$.
} 
Let

$$
\mathbf{E}=\mathcal{I}^{-}\left(\mathbf{M}^{(e n d)}\right) \cap \mathcal{I}^{+}\left(\mathbf{M}^{(e n d)}\right),
$$

where $\mathcal{I}^{-}\left(\mathbf{M}^{(e n d)}\right), \mathcal{I}^{+}\left(\mathbf{M}^{(e n d)}\right)$ denote the past and respectively future sets of $\mathbf{M}^{(e n d)}$. We assume that $\mathbf{E}$ is globally hyperbolic and

$$
\Sigma^{0} \cap \mathcal{I}^{-}\left(\mathbf{M}^{(e n d)}\right)=\Sigma^{0} \cap \mathcal{I}^{+}\left(\mathbf{M}^{(e n d)}\right)=\Phi_{0}\left(E_{1}\right) .
$$

We assume that $\mathbf{T}$ does not vanish at any point of $\mathbf{E}$ and that every orbit of $\mathbf{T}$ in $\mathbf{E}$ is complete and intersects transversally the hypersurface $\Sigma^{0} \cap \mathbf{E}$.

SBS. (Smooth bifurcation sphere assumption) It follows from (1.4) that

$$
\delta\left(\mathcal{I}^{-}\left(\mathbf{M}^{(e n d)}\right)\right) \cap \Sigma^{0}=\delta\left(\mathcal{I}^{+}\left(\mathbf{M}^{(e n d)}\right)\right) \cap \Sigma^{0}=S S_{0},
$$

where $S S_{0}=\Phi_{0}\left(\left\{x \in \mathbb{R}^{3}:|x|=1\right\}\right)$ is an imbedded 2-sphere (called the bifurcation sphere). We assume that there is a neighborhood $\mathbf{O}$ of $S S_{0}$ in $\mathbf{M}$ such that the sets

$$
\mathcal{H}^{+}=\mathbf{O} \cap \delta\left(\mathcal{I}^{-}\left(\mathbf{M}^{(e n d)}\right)\right) \quad \text { and } \quad \mathcal{H}^{-}=\mathbf{O} \cap \delta\left(\mathcal{I}^{+}\left(\mathbf{M}^{(e n d)}\right)\right)
$$

are smooth imbedded hypersurfaces. We assume that these hypersurfaces are null, nonexpanding 6 , and intersect transversally in $S S_{0}$. Finally, we assume that the vector-field $\mathbf{T}$ is tangent to both hypersurfaces $\mathcal{H}^{+}$and $\mathcal{H}^{-}$, and does not vanish identically on $S S_{0}$.

\subsubsection{Main Theorem.}

Theorem 1.1. Assume (M, g) is a regular black hole exterior satisfying the assumptions GR and SBS above, with a stationary Killing field T. Assume in addition that the hypersurface $\Sigma_{1}=\Phi_{0}\left(E_{1}\right)$ is maximal, and that

$$
\|\mathbf{g}(\mathbf{T}, \mathbf{T})\|_{L^{\infty}\left(S S_{0}\right)}<\epsilon^{2},
$$

where $\epsilon$ is a sufficiently small constant. Then $(M, \mathbf{g})$ is stationary and axially symmetric, thus, in view of the Carter-Robinson theorem [3, 16, 7], it is isometric to a Kerr solution with small angular momentum.

The proof of the theorem consists of two main steps: the first was done in our previous work [1] where we proved that there exists, locally in a small neighborhood of the event horizon, a second, rotational Killing vector field $\mathbf{Z}$ commuting with $\mathbf{T}$. More precisely our result in [1] can be stated as follows:

Theorem 1.2. Under the assumptions above, there exists an open set $\Omega \subset \mathbf{M}, \mathcal{S}_{0} \subseteq \Omega$, where $(M, \mathbf{g})$ admits a second rotational Killing vector-field $\mathbf{Z}$ which commutes with $\mathbf{T}$.

\footnotetext{
${ }^{6} \mathrm{~A}$ null hypersurface is said to be non-expanding if the trace of its null second fundamental form vanishes identically.

${ }^{7}$ See subsection 1.2 for a precise description of this smallness assumption.
} 
In step 2 we use ideas inspired from Sudarsky-Wald [17] to prove that that $\mathbf{T}$ becomes strictly timelike within the set $\Omega \cap \mathbf{E}$ and stays timelike throughout the complement of $\Omega$ in $\mathbf{E}$, provided that the constant $\epsilon$ in (1.5) is sufficiently small. Therefore, see [14, the space-time is analytic in a neighborhood of $\Sigma_{1}$, outside the domain $\Omega$. Therefore, using [15], the rotational Killing vector-field $\mathbf{Z}$ can be extended throughout the exterior region. Alternatively, we can avoid passing through real analyticity by observing that the $\mathbf{T}$ - conditional pseudo-convexity is automatically satisfied if $\mathbf{T}$ is time-like. We can therefore rely, as well, on the extension results proved in [10], [11], [2].

Finally, we can appeal to the Carter-Robinson theorem to conclude that $(\mathbf{M}, \mathbf{g})$ is isometric to a Kerr solution, with a small angular momentum $a=J / M$.

Therefore, the main goal of this paper is to show the following:

Proposition 1.3. Under the above assumptions there exists $\epsilon>0$ sufficiently small such that if (1.5) is satisfied then the ergoregion $\left\{p \in \Sigma_{1}: \mathbf{g}(\mathbf{T}, \mathbf{T}) \geq 0\right\}$ of $\mathbf{T}$ is contained in the domain $\Omega$ of extension of the rotational Killing field $\mathbf{Z}$ guaranteed by Theorem 1.2 .

1.2. The precise quantitative assumptions. The assumptions of the previous subsection were qualitative in nature. In this subsection we make them precise in a quantitative way to make sense of our smallness assumption (1.5).

Let $\partial_{1}, \partial_{2}, \partial_{3}$ denote the vectors tangent to $\Sigma^{0}$, induced by the diffeomorphism $\Phi_{0}$. Let $\Sigma_{r}=\Phi_{0}\left(E_{r}\right)$, where, as before, $E_{r}=\left\{x \in \mathbb{R}^{3}:|x|>r\right\}$. In particular, for our original spacelike hypersurface, we have $\Sigma^{0}=\Sigma_{1 / 2}$.

As in [2, Section 2.1], using (1.2) and the assumption that $\Sigma^{0}$ is spacelike, it follows that there are large constants $A_{1}$ and $R_{1} \geq R_{0}$, such that $R_{1} \geq A_{1}^{4}$, with the following properties: on $\Sigma_{3 / 4}$, for any $X=\left(X^{1}, X^{2}, X^{3}\right)$,

$$
A_{1}^{-1}|X|^{2} \leq \sum_{\alpha, \beta=1}^{3} X^{\alpha} X^{\beta} \mathbf{g}_{\alpha \beta} \leq A_{1}|X|^{2} \quad \text { and } \quad \sum_{\alpha=1}^{3}\left|\mathbf{g}\left(\partial_{\alpha}, \mathbf{T}\right)\right|+\left|\mathbf{g}\left(T_{0}, \mathbf{T}\right)\right| \leq A_{1} .
$$

In $\Phi_{0}\left(\mathbb{R} \times E_{R_{1}}\right)$, which we continue to denote by $\mathbf{M}^{(e n d)}, \mathbf{T}=\partial_{0}$ and (see notation in footnote 5 ,

$$
\begin{aligned}
\sum_{m=0}^{6} r^{m+1} \sum_{j, k=1}^{3}\left|\partial^{m}\left(\mathbf{g}_{j k}-\delta_{j k}\right)\right| & +\sum_{m=0}^{6} r^{m+2}\left|\partial^{m}\left(\mathbf{g}_{00}+1-2 M / r\right)\right| \\
& +\sum_{m=0}^{6} r^{m+3} \sum_{i=1}^{3}\left|\partial^{m}\left(\mathbf{g}_{0 i}+2 \epsilon_{i j k} S^{j} x^{k} r^{-3}\right)\right| \leq A_{1} .
\end{aligned}
$$

We construct a system of coordinates in a small neighborhood $\widetilde{\mathbf{M}}$ of $\Sigma^{0} \cap \overline{\mathbf{E}}$, which extends both the coordinate system of $\mathbf{M}^{(\text {end })}$ in (1.7) and that of $\Sigma^{0}$. We do that with the help of a smooth vector-field $T^{\prime}$ which interpolates between $\mathbf{T}$ and $T_{0}$. More precisely we construct $T^{\prime}$ in a neighborhood of $\Sigma_{3 / 4}$ such that $T^{\prime}=\mathbf{T}$ in $\Phi_{0}\left(\mathbb{R} \times E_{2 R_{1}}\right)$ and $T^{\prime}=$ $\eta\left(r / R_{1}\right) T_{0}+\left(1-\eta\left(r / R_{1}\right)\right) \mathbf{T}$ on $\Sigma_{3 / 4}$, where $\eta: \mathbb{R} \rightarrow[0,1]$ is a smooth function supported 
in $(-\infty, 2]$ and equal to 1 in $(-\infty, 1]$. Using now the flow induced by $T^{\prime}$ we extend the original diffeomorphism $\Phi_{0}: E_{1 / 2} \rightarrow \Sigma^{0}$, to cover a full neighborhood of $\Sigma_{1}$. Thus there exists $\varepsilon_{0}>0$ sufficiently small and a diffeomorphism $\Phi_{1}:\left(-\varepsilon_{0}, \varepsilon_{0}\right) \times E_{1-\varepsilon_{0}} \rightarrow \widetilde{\mathbf{M}}$, which agrees with $\Phi_{0}$ on $\{0\} \times E_{1-\varepsilon_{0}} \cup\left(-\varepsilon_{0}, \varepsilon_{0}\right) \times E_{2 R_{1}}$ and such that $\partial_{0}=\partial_{x^{0}}=T^{\prime}$.

By construction, using also (1.7) and letting $\varepsilon_{0}$ sufficiently small depending on $R_{1}$,

$$
\sum_{j=1}^{3}\left|\mathbf{g}_{0 j}\right|+\left|\mathbf{g}_{00}+1\right| \leq A_{1} /\left(R_{1}+r\right) \quad \text { in } \widetilde{\mathbf{M}} .
$$

Note, in particular, that the Killing field $T$ is time-like on $\Sigma_{R_{1}}$.

With $\mathbf{g}_{\alpha \beta}=\mathbf{g}\left(\partial_{\alpha}, \partial_{\beta}\right)$ and $\mathbf{T}=\mathbf{T}^{\alpha} \partial_{\alpha}$, let

$$
A_{2}=\sup _{p \in \widetilde{\mathbf{M}}} \sum_{m=0}^{6}\left[\sum_{\alpha, \beta=0}^{3}\left|\partial^{m} \mathbf{g}_{\alpha \beta}(p)\right|+\sum_{\alpha=0}^{3}\left|\partial^{m} \mathbf{T}^{\alpha}(p)\right|\right] .
$$

Finally, we fix

$$
\bar{A}=\max \left(R_{1}, A_{2}, \varepsilon_{0}^{-1},\left(M^{2}-J\right)^{-1}\right) .
$$

The constant $\bar{A}$ is our main effective constant. Notice that this constant also controls the components of the contra-variant metric $\mathbf{g}^{\alpha \beta}$ (and their derivatives), as a consequence of (1.6) and (1.9). The constant also controls the components of the second fundamental form $k$ (see (2.1) ) along $\Sigma^{0}$, and their derivatives 8 .

The constant $\epsilon$ in (1.5) will be assumed sufficiently small, depending only on $\bar{A}$. Throughout the remaining paper we use the notation $A \lesssim B$ to denote unequalities $A \leq C B$, with a universal constant $C>0$ which depends only on $\bar{A}$. Similarly $A \gtrsim B$ means $A \geq C B$.

To summarize, we have defined a neighborhood $\widetilde{\mathbf{M}}$ of $\Sigma^{0} \cap \overline{\mathbf{E}}$ and a diffeomorphism $\Phi_{1}:\left(-\varepsilon_{0}, \varepsilon_{0}\right) \times E_{1-\varepsilon_{0}} \rightarrow \widetilde{\mathbf{M}}, \varepsilon_{0}>0$, such that the bounds (1.6), (1.7), (1.8), (1.9) hold (in coordinates induced by the diffeomorphism $\Phi_{1}$ ).

\section{Proof OF THE MAIN THEOREM}

2.1. Some useful identities. In this subsection we gather various formulas relating the Killing vector-field $\mathbf{T}$, the metric $\mathbf{g}$, and the maximal hypersurface $\Sigma_{1}$. Let $h:=\left.\mathbf{g}\right|_{\Sigma^{0}}$ denote the induced metric on the hypersurface $\Sigma^{0}$ and let $\nabla$ denote the induced LeviCivita connection. Also let $T_{0}$ denote the future unit normal vector-field to $\Sigma^{0}$. Let $k_{i j}$ denote the second fundamental form of the hypersurface $\Sigma^{0}$,

$$
k(Y, Z):=-\mathbf{g}\left(\mathbf{D}_{Y} T_{0}, Z\right),
$$

for all vector-fields $Y, Z$ tangent to $\Sigma^{0}$. Notice that

$$
\nabla_{Y} Z=\mathbf{D}_{Y} Z+k(Y, Z) T_{0},
$$

\footnotetext{
${ }^{8}$ We remark that the constant $\bar{A}$ depends only on $M+1 / M+1 /(M-a)$ in the case when $\mathbf{E}$ is isometric to the domain of outer communications of the Kerr space-time $\mathcal{K}(M, a)$. It does not increase when $a$ approaches 0 , if $M$ is fixed.
} 
for all vector-fields $Y, Z$ tangent to $\Sigma^{0}$. We also recall the Gauss equation,

$$
\nabla_{i} k_{j m}-\nabla_{j} k_{i m}=-\mathbf{R}_{i j m \alpha}\left(T_{0}\right)^{\alpha} .
$$

In our case, since $\Sigma_{1}$ is a maximal hypersurface we have, by definition,

$$
h^{i j} k_{i j}=0 \text {. }
$$

Using also the Gauss equation and the Einstein vacuum equations it follows that

$$
\nabla^{i} k_{i j}=0 \text {. }
$$

We now turn to the a natural decomposition of the Killing vector $\mathbf{T}$ relative to our hypersurface,

$$
\mathbf{T}=n T_{0}+X
$$

where $n=-\mathbf{g}\left(\mathbf{T}, T_{0}\right)$ (the lapse function) is a smooth real-valued function on $\Sigma^{0}$, and $X \in T \Sigma^{0}$ (the shift vector) is a smooth vector-field along $\Sigma^{0}$ that satisfies $\mathbf{g}\left(X, T_{0}\right)=0$. Since $\mathbf{T}$ is Killing, it follows easily from (2.2) and the decomposition (2.6) that

$$
\nabla_{i} X_{j}+\nabla_{j} X_{i}=2 n k_{i j}
$$

Finally, the Killing equation together with the maximality imply that $k_{i j}, n$ satisfy the lapse equation

$$
\Delta n=|k|^{2} n \quad \text { along } \Sigma_{1},
$$

where $\Delta:=\nabla^{i} \nabla_{i}$ is the Laplace-Beltrami operator induced on the surface $\Sigma^{0}$.

2.2. Proof of Proposition 1.3. We show first how to control the lapse function $n$ along the surface $\Sigma_{1}$.

Lemma 2.1. The function $n$ satisfies

$$
n(p) \in[0,1) \quad \text { for any } p \in \Sigma_{1} .
$$

Moreover, there is a constant $C_{1}$ that depends only on the constant $\bar{A}$ in (1.10) such that

$$
n(p) \geq C_{1}^{-1} \frac{r(p)-1}{r(p)} \quad \text { for any } p \in \Sigma_{1} \text {. }
$$

Proof of Lemma 2.1. The identity $\mathbf{T}=n T_{0}+\sum_{j=1}^{3} X^{j} \partial_{j}$ together with the asymptotic flatness assumption (1.2) show that

$$
X^{1}, X^{2}, X^{3}=O_{6}\left(r^{-2}\right) \quad \text { and } \quad n=1-M / r+O_{6}\left(r^{-2}\right) \quad \text { in } \Sigma_{R_{1}} .
$$

Moreover, $n \equiv 0$ on $\mathcal{S}_{0}$, since $\mathbf{T}$ is tangent to $\mathcal{S}_{0}$. Recall also that $n$ satisfies the elliptic equation $\Delta n=|k|^{2} n$ along $\Sigma_{1}$, see (2.8).

The bound (2.9) follows as a consequence of the weak maximum principle applied to the function $n$ in the domain $\Sigma_{1} \backslash \overline{\Sigma_{R_{1}}}$. The bound (2.10) follows from the proof of the strong maximum principle (Hopf lemma), see, for example, [8, Chapters 3.1, 3.2].

We use now our main assumption (1.5) to show that $k$ is small along $\Sigma_{1}$. 
Lemma 2.2. For any $i, j \in\{1,2,3\}$ we have

$$
\left\|k_{i j}\right\|_{L^{\infty}\left(\Sigma_{1}\right)} \leq \epsilon^{1 / 8}
$$

Proof of Lemma 2.2. We combine the identities (2.5) and (2.7) to derive the formula

$$
n|k|^{2}=k^{i j} n k_{i j}=\nabla^{i} X^{j} k_{i j}=\nabla^{i}\left(X^{j} k_{i j}\right),
$$

along $\Sigma_{1}$. Since $X=\mathbf{T}$ along $\mathcal{S}_{0}$, it follows from (1.5) that

$$
\sum_{i=1}^{3}\left\|X^{j} k_{i j}\right\|_{L^{\infty}\left(\mathcal{S}_{0}\right)} \lesssim \epsilon .
$$

Moreover, using the asymptotic flatness assumption (1.2) and the definitions it is easy to see that

$$
k_{i j}=O_{6}\left(r^{-2}\right), \quad \text { in } \Sigma_{R_{1}} \text { for any } i, j \in\{1,2,3\} .
$$

Therefore, we can integrate by parts along the surface $\Sigma_{1}$ to conclude that

$$
\int_{\Sigma_{1}} n|k|^{2} d \mu \lesssim \epsilon
$$

We can now prove the pointwise bound (2.12). Assume, for contradiction, that $\left|k_{i j}(p)\right| \geq$ $\epsilon^{1 / 8}$ for some $i, j \in\{1,2,3\}$ and $p \in \Sigma_{1}$. In view of the smoothness assumption, it follows that there is a constant $C=C(\bar{A})$ sufficiently large such that $\left|k_{i j}\left(p^{\prime}\right)\right| \geq \epsilon^{1 / 8} / 2$ for all points $p^{\prime} \in B(p):=\left\{p^{\prime} \in \Sigma_{1}:\left|p-p^{\prime}\right| \leq C^{-1} \epsilon^{1 / 8}\right\}$. In addition, using (2.10),

$$
\int_{B(p)} n d \mu \gtrsim \epsilon^{4 / 8}
$$

Therefore

$$
\int_{B(p)} n|k|^{2} d \mu \gtrsim \epsilon^{6 / 8}
$$

which contradicts (2.14). This proves the desired pointwise bound (2.12).

Finally, we show that $X$ stays small along the surface $\Sigma_{1}$.

Lemma 2.3. We have

$$
\sup _{p \in \Sigma_{1} \backslash \Sigma_{R_{1}}}\left[\sum_{j=1}^{3}\left|X_{j}(p)\right|+\sum_{i, j=1}^{3}\left|\nabla_{i} X_{j}(p)\right|\right] \leq \epsilon^{1 / 30} .
$$

Proof of Lemma 2.3. We show first that

$$
\left\|\nabla_{l} k_{i j}\right\|_{L^{\infty}\left(\Sigma_{1}\right)} \leq \epsilon^{1 / 20}
$$

for any $l, i . j \in\{1,2,3\}$. Indeed, assume for contradiction that $\left|\nabla_{l} k_{i j}(p)\right| \geq \epsilon^{1 / 20}$ for some point $p \in \Sigma_{1}$. Then, using the smoothness assumption and the bound (2.12), it follows that $\left|\partial_{l} k_{i j}\left(p^{\prime}\right)\right| \geq \epsilon^{1 / 20} / 2$ for all points $p^{\prime} \in \Sigma_{1}$ with the property that $\left|p^{\prime}-p\right| \leq$ $\epsilon^{1 / 18}$. Therefore there is a point $p^{\prime} \in \Sigma_{1}$ with the property that $\left|p^{\prime}-p\right| \leq \epsilon^{1 / 18}$ and $\left|k_{i j}\left(p^{\prime}\right)-k_{i j}(p)\right| \geq \epsilon^{1 / 9}$. This contradicts the bound (2.12). 
The vector-field $X$ satisfies the approximate Killing equation

$$
\nabla_{i} X_{j}+\nabla_{j} X_{i}=2 n k_{i j}
$$

Recall also that $\left|X_{j}(p)\right| \lesssim \epsilon$ for $p \in \mathcal{S}_{0}$ and $j \in\{1,2,3\}$. The same interpolation argument as above shows that

$$
\left\|h\left(\nabla_{V} X, \partial_{j}\right)\right\|_{L^{\infty}\left(\mathcal{S}_{0}\right)} \lesssim \epsilon^{1 / 3},
$$

for any $j \in\{1,2,3\}$ and any vector-field $V=V^{1} \partial_{1}+V^{2} \partial_{2}+V^{3} \partial_{3}$ tangent to the bifurcation sphere $\mathcal{S}_{0}$, satisfying $\sum_{i=1}^{3}\left\|V^{i}\right\|_{L^{\infty}\left(\mathcal{S}_{0}\right)} \leq 1$. Moreover, using (2.17),

$$
h\left(\nabla_{W} X, W\right)=0 \quad \text { on } \mathcal{S}_{0},
$$

since $n$ vanishes along $\mathcal{S}_{0}$. Combining with (2.18) it follows that

$$
\left\|h\left(\nabla_{\partial_{i}} X, \partial_{j}\right)\right\|_{L^{\infty}\left(\mathcal{S}_{0}\right)} \lesssim \epsilon^{1 / 3},
$$

for any $i, j \in\{1,2,3\}$. Therefore

$$
\sum_{j=1}^{3}\left\|X_{j}\right\|_{L^{\infty}\left(\mathcal{S}_{0}\right)}+\sum_{i, j=1}^{3}\left\|\nabla_{i} X_{j}\right\|_{L^{\infty}\left(\mathcal{S}_{0}\right)} \leq \epsilon^{1 / 4} .
$$

To prove the desired estimate (2.15) we need to extend the inequality (2.19) from the bifurcation sphere $\mathcal{S}_{0}$ to the region $\Sigma_{1} \backslash \Sigma_{R_{1}}$. We use the equation (2.17), which is an approximate Killing equation for $X$ along $\Sigma_{1}$. The argument we present below is a quantitative version of the well-known argument showing that a Killing vector-field vanishes identically in a connected open set if it vanishes up to order 1 at one point.

More precisely, let

$$
\pi_{i j}:=\nabla_{i} X_{j}+\nabla_{j} X_{i}=2 n k_{i j}
$$

and recall the general formula

$$
\nabla_{a} \nabla_{b} X_{c}=X^{d} R_{d a b c}+(1 / 2)\left(\nabla_{a} \pi_{b c}+\nabla_{b} \pi_{a c}-\nabla_{c} \pi_{a b}\right) .
$$

Therefore, in view of (2.12) and (2.16),

$$
\left\|\nabla_{l} \nabla_{i} X_{j}-X^{d} R_{d l i j}\right\|_{L^{\infty}\left(\Sigma_{1} \backslash \Sigma_{R_{1}}\right)} \lesssim \epsilon^{1 / 20} .
$$

Assume now that $p=\Phi_{0}\left(r_{0} \omega\right)$ is a point in $\Sigma_{1} \backslash \Sigma_{R_{1}}, r_{0} \in\left[1, R_{1}\right], \omega \in \mathbb{S}^{2}$. Let $p^{\prime}=\Phi_{0}(\omega) \in \mathcal{S}_{0}$ and $\gamma:[0,1] \rightarrow \Sigma_{1} \backslash \Sigma_{R_{1}}, \gamma(t)=\Phi_{0}\left[\left(1+\left(r_{0}-1\right) t\right) \omega\right]$ denote a curve connecting the points $p^{\prime}$ and $p$. Let $V(t)=\dot{\gamma}(t)$ denote the vector-field tangent along the curve $\gamma$. In view of (2.20), the functions $\nabla_{i} X_{j}$ and $X_{j}$ satisfy the system of transport equations

$$
\begin{aligned}
& \nabla_{V} X_{j}-V^{i} \nabla_{i} X_{j}=0 \\
& \left\|\nabla_{V} \nabla_{i} X_{j}-X_{d} V_{l} R^{d l}{ }_{i j}\right\|_{L^{\infty}} \lesssim \epsilon^{1 / 20} .
\end{aligned}
$$

along the curve $\gamma$. The desired bound (2.15) follows using also (2.19).

We can now complete the proof of Proposition 1.3 
Proof of Proposition 1.3. The formula $\mathbf{T}=n T_{0}+X$ shows that

$$
\mathbf{g}(\mathbf{T}, \mathbf{T})=-n^{2}+h(X, X) \quad \text { along } \Sigma_{1}
$$

Using (2.10) and (2.15) it follows that

$$
\mathbf{g}(\mathbf{T}, \mathbf{T}) \leq-\epsilon \quad \text { in } \Sigma_{1+\epsilon^{1 / 100}}
$$

On the other hand, the main theorem in [1] guarantees the existence of a rotational Killing vector-field $\mathbf{Z}$ in a region $\Omega$, which contains the set $\Sigma_{1} \backslash \Sigma_{1+\rho}$ for some constant $\rho=\rho(\bar{A})>0$. The conclusion of the proposition follows.

\section{REFERENCES}

[1] S. Alexakis, A. D. Ionescu, and S. Klainerman, Hawking's local rigidity theorem without analyticity, Geom. Funct. Anal. 20 (2010), 845-869.

[2] S. Alexakis, A. D. Ionescu, and S. Klainerman, Uniqueness of smooth stationary black holes in vacuum: small perturbations of the Kerr spaces, Commun. Math. Phys. 299 (2010), 89-127.

[3] B. Carter, An axy-symmetric black hole has only two degrees of freedom, Phys. Rev. Lett. 26 (1971), 331-333.

[4] B. Carter, Has the Black Hole equilibrium problem been solved ?, arXiv preprint gr-qc/9712038, 1997 - arxiv.org

[5] D. Christodoulou and S. Klainerman, The global nonlinear stability of the Minkowski space, Princeton Math. Series 41, Princeton University Press (1993).

[6] P. T. Chrusciel and R. M. Wald, Maximal hypersurfaces in stationary asymptotically flat spacetimes, Comm. Math. Phys. 163 (1994), 561-604.

[7] P. T. Chrusciel and J. L. Costa, On uniqueness of stationary vacuum black holes, Astérisque 321 (2008), 195-265.

[8] D. Gilbarg and N. Trudinger, Elliptic partial differential equations of second order, Reprint of the 1998 edition, Classics in Mathematics, Springer-Verlag, Berlin, (2001).

[9] S. W. Hawking and G. F. R. Ellis, The large scale structure of space-time, Cambridge Univ. Press, (1973).

[10] A. D. Ionescu and S. Klainerman, On the uniqueness of smooth, stationary black holes in vacuum, Invent. Math. 175 (2009), 35-102.

[11] A. D. Ionescu and S. Klainerman, Uniqueness results for ill-posed characteristic problems in curved space-times, Commun. Math. Phys. 285 (2009), 873-900.

[12] A. D. Ionescu and S. Klainerman, On the local extension of Killing vector-fields in Ricci flat manifolds, to appear on J.A.M.S.

[13] W. Israel, Event horizons in static vacuum space-times, Phys. Rev. Letters 164 (1967), 1776-1779.

[14] H. Müller zum Hagen, On the analyticity of static vacuum solutions of Einstein's equations, Proc. Cambridge Philos. Soc. 67 (1970), 415-421.

[15] K. Nomizu, On local and global existence of Killing vector fields, Ann. of Math. 72 (1960), 105-120.

[16] D.C. Robinson, Uniqueness of the Kerr black hole, Phys. Rev. Lett. 34 (1975), 905-906.

[17] D. Sudarski and R. M. Wald, Mass formulas for stationary Einstein-Yang-Mills black holes and a simple proof of two staticity theorems, Phys. Rev. D47 (1993), R5209-R5213. 
UNIVERSITY OF TORONTO

E-mail address: alexakis@math.toronto.edu

Princeton University

E-mail address: aionescu@math.princeton.edu

Princeton University

E-mail address: seri@math.princeton.edu 